\title{
Una aproximación a la historiografía urbana: algunos aspectos epistemológicos y metodológicos
}

Izaskun Landa. Universidad Central de Venezuela, Caracas, Venezuela.

RESUMEN | El propósito de este ensayo es explorar ciertos aspectos epistemológicos y metodológicos de la historiografía urbana. En lo epistemológico se establece una breve aproximación a la historiografía urbana, luego se analizan y se presentan ejemplos sobre tres formas de conocimiento del pasado urbano: la descripción, la comprensión y la explicación, y se trazan algunos de los paradigmas de la historiografía urbana occidental y de la latinoamericana. En el aparte metodológico se aborda el tema de las prácticas de investigación de la historiografía urbana a través del examen del método histórico, y se presentan las características y ejemplos de los procesos empleados por ciertos historiadores urbanos. Por último, se aborda el tema del tiempo y de las periodizaciones, tanto de la historiografía urbana occidental como de la latinoamericana en particular.

PALABRAS CLAVE | historia urbana, teoría urbana, urbanismo.

ABSTRACT | The purpose of this essay is to present a brief analysis about some epistemological and methodological aspects of the urban historiography, through the examination offew of the Occidental and Latin American main works. The epistemological section contains first, a brief approximation to urban history epistemology, second, the analysis of main forms of urban historical knowledge: such as description, understanding and explanation, and third, a selection of some important paradigms or approaches of the Occidental and Latin American urban historiography. The methodological section describes and explains the historical method proceedings. Finally, concepts and classifications of time and some Occidental and Latin American periodizations of urban history are presented.

KEYwORDs | urban history, urban theory, urbanism. 


\section{Introducción}

El presente ensayo nace de la necesidad de explorar algunos elementos epistemológicos y metodológicos que hasta ahora no han sido explícitamente considerados en los escasos textos escritos sobre los fundamentos de la historiografía urbana. Estas obras hacen énfasis principalmente en las aproximaciones epistemológicas del saber histórico urbano, de manera que en este opúsculo se abren otros aspectos para ser explorados, tales como las formas del conocimiento y las operaciones del método histórico empleadas en el estudio del fenómeno urbano en la historia.

A este respecto y a partir de un análisis novedoso, se pretende profundizar y ampliar la comprensión acerca de la naturaleza y los fundamentos constituyentes del saber histórico sobre la ciudad y sus procesos.

En este ensayo se aborda el tema de la historiografía urbana desde la experiencia hermenéutica, como comprensión e interpretación del texto por parte del sujeto en tanto unidad entre la realidad histórica y su comprensión, donde las interpretaciones "fenomenistas" y "objetivistas" de la realidad histórica se complementan mutuamente (Gadamer, 1993). En este sentido de fusión de visiones, pero en el plano epistemológico, se asume el estudio de la historiografía en cuanto concepción y métodos de manera amplia y no restrictiva, al incorporar en ciertos casos el saber científico al de las ciencias humanas y las sociales; de lo contrario, se estaría limitando el universo de las obras consultadas, y ahí radica uno de los aportes del presente ensayo. Lo anterior permite entender la razón por la cual los textos objeto de este estudio son de naturaleza diversa en tanto conocimiento preponderantemente humanístico, pero también de las ciencias sociales y las exactas.

En un sentido distinto y en otro nivel de análisis, se tiene, por una parte, la referencia a ciertas obras de carácter metodológico y epistemológico sobre la historia general, alusión que resulta fundamental en la interpretación de los textos de historia urbana, pues permite explorar las formas del conocimiento y los procedimientos metodológicos de la historia urbana, y de ahí proviene otro de los aportes de este ensayo. Por otra parte, se encuentra un grupo de textos destacados de la historiografía urbana occidental y latinoamericana referidos a dilatados periodos históricos, de donde proviene la fortaleza de este trabajo en tanto visión comprehensiva y diacrónica de los fenómenos urbanos en el tiempo. No obstante, esta especificidad constituye también su principal limitación, ya que los copiosos estudios de caso de historia urbana no han sido objeto de la presente revisión.

Tanto los fundamentos epistemológicos analizados a través de las formas del conocimiento y los paradigmas interpretativos, como el tema del tiempo y las periodizaciones, noción crucial de la historia, son categorías conceptuales y aspectos consustanciales a la epistemología de la historia urbana, aunque su entendimiento requiere de la separación analítica, y así son presentados a continuación. Los procedimientos metodológicos y alguna de sus diversas operaciones, por otra parte, también están íntimamente entrelazados con las formas del conocimiento, como se expone en la sección correspondiente. 


\section{Algunos elementos epistemológicos de la historiografía urbana}

Esta investigación parte necesariamente de la pregunta sobre qué es la historiografía urbana. Posiblemente, Almandoz (2008, p. 22) es uno de los pocos autores que la ha abordado, y la define como el conjunto de obras o textos que se refieren a la historia de la ciudad, la urbanización y el urbanismo, y a su estudio. Estas obras presentan los objetos tradicionales de estudio, como son la ciudad y los procesos de urbanización, pero, además, los amplían a la historia del urbanismo como disciplina técnica, tema fundamental de numerosos estudios historiográficos a partir de los años sesenta del siglo xx. La historia urbana, por otra parte, trata sobre los acontecimientos o hechos ocurridos en el pasado (Le Goff, 1991), referidos a la ciudad, la urbanización y el urbanismo.

En cuanto al concepto de ciudad, en el caso de esta investigación abarca un amplio conjunto de asentamientos urbanos, que comprenden desde las pequeñas ciudades de los inicios urbanos hasta las extensas aglomeraciones metropolitanas de hoy, como lo expresan autores fundamentales mencionados en este estudio.

Ahora bien, la naturaleza de la ciudad y de sus procesos ha sido establecida desde distintas perspectivas que, por una parte, resaltan aspectos particulares, como el carácter demográfico (Capel, 1975; Germani, 1976), socioeconómico (Sjoberg, 1976; Weber, 1958; Wirth, 1968), o morfológico (Capel, 2002); y por otra, los estudian en su condición de entramado diverso (Mumford, 1937). Esta heterogeneidad de enfoques destaca la variedad y complejidad como características fundamentales de la historiografía urbana. Asimismo, resalta la diversidad temática y metodológica que se expresa tanto desde ópticas integrales como desde las visiones sectoriales de diferentes competencias disciplinarias: la historia, la sociología, la arquitectura, el urbanismo, el derecho, la antropología, la medicina sanitaria, la economía, la demografía, la geografía o la literatura. De manera que, como considera Terán (1996), la heterogeneidad y la fragmentariedad parecen ser las características comunes del saber histórico urbano, debido a la diversidad de las aportaciones disciplinares, y esta es la razón por la que las aproximaciones metodológicas que emplean los historiadores urbanos son múltiples y han sido calificadas de "eclécticas" por Guardia, Monclús y Oyón (1996).

Estas apreciaciones nos llevan a inferir que la coherencia de la historiografía urbana como área del saber estribaría principalmente en la concurrencia de los distintos enfoques disciplinares en el análisis de la ciudad, la urbanización y el urbanismo, como objetos comunes de estudio; sin embargo, es fundamental advertir que "lo urbano", como objeto unificador de la historiografía urbana es un término excesivamente general e indefinido, según ha señalado el reconocido historiador urbano Fernando de Terán (1996). No es objeto de esta monografía dilucidarlo, de manera que queda para investigadores interesados tanto definir su núcleo fundamental a partir de los estudios tradicionales, como establecer las zonas periféricas desde perspectivas novedosas.

Otro aspecto que debe señalarse es que desde los años sesenta del siglo $\mathrm{xx}$, la creciente y reconocida comunidad de historiadores urbanos ha incrementado el corpus de la historia urbana a través de la producción de investigaciones publicadas 
en revistas especializadas y en memorias de eventos nacionales e internacionales. Ellas nutren los programas de las nuevas cátedras de historia urbana creadas en universidades e institutos de investigación. Por estos motivos, reconocidos estudiosos como Almandoz y González Casas (1996), y Sambricio (1996), opinan que la historia urbana es "una disciplina académica". Sin embargo, esta posición no es compartida por importantes historiadores, entre ellos Veyne (1984), quien considera la historia de la ciudad como un área temática de la historia.

Una vez realizadas estas consideraciones, en esta primera parte de la investigación se abordan dos aspectos epistemológicos fundamentales, como son las formas del conocimiento y las aproximaciones o paradigmas interpretativos de la historiografía urbana.

\section{Las formas del conocimiento en la historiografía urbana}

Uno de los aspectos fundamentales de cualquier disciplina es determinar cuáles son las formas del conocimiento utilizadas para comprender y representar su objeto de estudio, aunque en el caso de la historiografía urbana ningún investigador parece haber explorado este tema. Como se ha señalado anteriormente, la historia urbana es un área del saber en la que concurren numerosas disciplinas, de manera que, para establecer sus formas de conocimiento, se parte de la posición de autores provenientes de la historia y de las ciencias sociales, como Bauer (1957) y Singleton, Straits y Miller (1993), respectivamente, quienes han señalado que los fenómenos del pasado y los presentes se hacen inteligibles mediante la descripción, la comprensión y la explicación. A partir del análisis de estos tres conceptos y de ejemplos provenientes de conocidas obras sobre la historia urbana, a continuación se intenta construir una visión inédita acerca de uno de los fundamentos sobre los que se pretende comprender la naturaleza del saber histórico urbano.

1. En primer lugar, la descripción de las características de las ciudades y de los fenómenos que en ellas ocurren en el tiempo es, posiblemente, la forma más utilizada para representar el fenómeno urbano en la historia. A partir del análisis de diferentes obras sobre la ciudad en la historia, es posible hacer dos distinciones: por un lado se encuentran las descripciones genéticas, que dan cuenta del origen y transformaciones de un grupo de ciudades o de una determinada ciudad; ejemplos de ello son el estudio de Pirenne (1972) acerca de las ciudades medievales, o el de Gasparini y Posani (1969) sobre la ciudad de Caracas. Otra de las formas descriptivas más utilizadas en la historiografía urbana es la construcción de modelos de ciudad; tal es el caso de los modelos urbanos de la escuela de Chicago (Park, 1967), de los planteados por Hardoy (1972) sobre las ciudades latinoamericanas, o los modelos que son el producto de las civilizaciones humanas elaborados por Weber (1958) o Mumford (1966), entre otros. Sin embargo, en numerosas ocasiones, los investigadores explican los procesos urbanos combinando las descripciones genéticas con los modelos de ciudad. Es el caso del arquitecto Benévolo (1978, vol. 2, p. 5), quien considera que su obra sobre la historia de la ciudad occidental está fundamentada en modelos históricos de ciudad que explican fenómenos con base en categorías de orden 
económico, político, social y cultural. Dos ejemplos esenciales concernientes al ámbito latinoamericano son los trabajos de Hardoy (1972) y Mejía Pavony (2013), quienes presentan análisis históricos de la urbanización latinoamericana a partir de interpretaciones genéticas que establecen el origen y las etapas de conformación de las ciudades y el territorio latinoamericano.

Algunas de las características del conocimiento por descripción se refieren al lenguaje técnico y los conceptos propios de las disciplinas que estudian la ciudad, ya que permiten representar y concebir los fenómenos urbanos. Estos conceptos pueden ser sencillos y remitir a lo concreto, como, por ejemplo, el de espacio urbano; o más complejos, como el de estructura urbana o el de tejido urbano, que incluyen diversos elementos de orden social, económico, espacial y constructivo (Capel, 2002). De esta forma, el lenguaje técnico y los conceptos empleados en las descripciones constituyen una de las bases del entendimiento de la historia urbana. Sin embargo, generalmente la descripción es insuficiente para analizar la ciudad y los fenómenos urbanos en la historia, de manera que, incluso en obras menos complejas sobre los procesos urbanos, se hace uso de la comprensión.

2. En cuanto a la comprensión como forma de conocimiento, ella está asociada, por una parte, a los procesos causales; y por otra, a construcciones conceptuales llamadas, o no, teorías (Hernández, Fernández, \& Baptista 1998), que a veces se emplean en historia urbana. Es posible que las explicaciones más comunes y fundamentales en la historiografía urbana sean el resultado del análisis de procesos que relacionan acontecimientos, asignándoles una condición causal. Algunos historiadores, como Carr (1982), plantean que el estudio de la historia es el examen de las causas y la selección de las más significativas. En relación con ello, Aróstegui (2001) considera que "explicar (...) es, intrínsecamente, argumentar por qué un estado social se transforma en otro” (p. 299). Al respecto, Chueca (1980) presenta el ejemplo, entre otros, del proceso de crecimiento urbano comprendido como resultado del asiento de las instituciones del poder y de la administración pública en determinados poblados; así también, como consecuencia del incremento de las actividades económicas. El decrecimiento urbano puede ser la consecuencia de fenómenos naturales, o de las guerras y la violencia.

Dado que las causas de los procesos urbanos son de diversa naturaleza y complejidad, la clasificación planteada por Topolsky (1992) permite comprender la magnitud y características de los fenómenos urbanos en el tiempo. Por una parte están las acciones humanas destinadas a un fin, definidas como acciones racionales emprendidas por individuos y grupos sociales que tienen un objetivo. Ejemplo de ello son el establecimiento de normas y procedimientos para el control de las obras públicas o la aplicación de nuevos modelos urbanos, como los planteados por los tratadistas del Renacimiento, cuyos preceptos transformaron el trazado y la estética urbana, según lo describe Morris (1995), entre otros autores. También está la comprensión de las consecuencias impensadas de acciones emprendidas por mucha gente, llamada por Topolsky (1992, p. 420) la 
comprensión de "los procesos". Refieren estos, por ejemplo, al proceso de urbanización de la humanidad que comienza hace 5500 años; también al de urbanización como consecuencia de la industrialización iniciada en el siglo XVIII, que en algunos países de Latinoamérica tiene sus inicios en la tercera década del siglo xx y ha sido registrado estadísticamente, y del cual se han establecido correlaciones como las de la Cepal (Comisión Económica para América Latina y el Caribe, 1976). Numerosos ejemplos para sustentar el entendimiento de la realidad histórica urbana a partir de la explicación causal pueden ańadirse a los presentados en este aparte.

A diferencia de la causalidad, otra de las formas del conocimiento por comprensión está principalmente asociada a la aplicación de teorías (Singleton et al., 1993), construcciones empleadas por los científicos sociales aunque también aceptadas por reconocidos metodólogos de la historia, entre ellos Topolsky (1992, pp. 287-291). ${ }^{1}$ Varias de tales formulaciones teóricas han sido utilizadas como marco de ideas para el análisis de la ciudad y los procesos que en ella ocurren; por ejemplo, la teoría de las permanencias de Poëte (Rossi, 1982) en el plano morfológico, las llamadas teorías europea y americana de la ciudad, de Martindale y Newirth (1958), en tanto que en el ámbito latinoamericano se pueden mencionar la teoría de la urbanización dependiente y la teoría de la marginalidad (Jaramillo, 1989-1990). No obstante, esta forma de comprensión es menos común que la causal.

La forma de conocimiento por comprensión parece abarcar gran parte de la literatura histórica urbana, pero deja de lado obras reconocidas que, aunque menos frecuentes, se fundamentan en hechos y patrones pasados y presentes, a partir de los cuales es posible establecer empíricamente escenarios futuros.

3. Por último, la explicación científica en historia es materia de discusión y controversia, ya que la historia no predice; empero, es insoslayable considerar la explicación a partir de principios abstractos, debido a la existencia de obras que los emplean. En este sentido, Veyne (1984), uno de los epistemólogos fundamentales de la historia en el siglo pasado, da por cierto que el historiador "hace uso de algunas verdades científicas, principalmente en materia económica y demográfica” (p. 69). Es el caso de la demografía histórica, empleada en el análisis de la dinámica y composición poblacional de las ciudades en determinados lapsos históricos; ejemplo de ello son los estudios de Negrón (2001) sobre el crecimiento de las ciudades venezolanas desde la explotación petrolera.

Asimismo, investigadores de la escuela de economía neoclásica urbana han formulado teorías de la renta de la tierra y de la localización (Palacios, 1980) que utilizan modelos matemáticos en el marco de un sistema hipotético-deductivo, para explicar y simular algunos fenómenos urbanos; por ejemplo, el proceso de localización de actividades urbanas y de flujos entre ellas, incorporado por Alonso (1965) en su estudio. Estas teorías no son ahistóricas, como ocurre con

$1 \longdiv { \text { En historia, las teorías son series de afirmaciones estrictamente históricas que permiten reconstruir } }$ diversos fragmentos del pasado, al margen de su lugar y su papel en la estructura entera del pasado, como por ejemplo el origen y la formación de ciudades. 
las leyes físicas, ya que generalmente se aplican a partir del surgimiento de la ciudad moderna capitalista. Sin embargo, este tipo de explicación es poco utilizada en las investigaciones de historia urbana.

Estas tres formas de inteligibilidad de la historia urbana son empleadas, en mayor o menor medida, para interpretar lo urbano en el tiempo, según cada autor, de acuerdo con su proveniencia disciplinaria o la tradición particular asumida, aunque a veces se adoptan de manera independiente a ellas. Estas formas del conocimiento se encuentran también fusionadas con los procedimientos metodológicos, como se puede observar en el ejemplo de la aplicación, o no, de teorías y construcciones conceptuales mostrado en el aparte correspondiente. En síntesis, a través de ciertos ejemplos ilustrativos ha sido posible establecer que la historiografía urbana hace inteligibles las características de la ciudad y de los procesos urbanos en el pasado, mediante distintos recursos: la descripción y los modelos; la comprensión causal que utiliza, o no, teorías; y la explicación científica, cuando aplica los conocimientos demográficos y de economía.

De aquí se desprende que las formas del conocimiento empleadas en la historiografía urbana permiten interpretar la naturaleza compleja de sus objetos de estudio, bien sea la ciudad, el proceso de urbanización y el urbanismo como disciplina, lo que además demuestra el carácter esencialmente heterogéneo y múltiple de este subcampo del conocimiento.

\section{Los paradigmas interpretativos de la investigación en la historiografía urbana}

Toda investigación sobre la historia urbana, de cualquier tipo que sea, parte de una aproximación particular en tanto modelo de interpretación del fenómeno urbano en el tiempo. En este sentido, Kuhn (2004) define los paradigmas como patrones aceptados de investigación científica de los cuales surgen tradiciones particulares y coherentes, las cuales sirven de guía para definir los problemas, métodos y soluciones de investigación a una comunidad de profesionales.

En el caso de los textos de historia urbana es necesario partir de la obra del urbanista Almandoz (2008), quien ha podido trazar y establecer el origen, desarrollo y fundamentos de numerosas aproximaciones epistemológicas y tradiciones particulares, tanto europeas como norteamericanas y latinoamericanas, en la medida en que el urbanismo iba constituyéndose como disciplina. Por otra parte, los arquitectos Guardia, Monclús y Oyón (1996) analizan someramente diversas aproximaciones epistemológicas pioneras de las tradiciones morfológicas y del paisaje urbano en Europa y los Estados Unidos, y consideran el enfoque socioespacial como uno de los núcleos distintivos de la historiografía urbana. A partir de la obra de los mencionados autores, se presentan a continuación algunas corrientes ilustrativas:

- Las aproximaciones que se fundamentan en la dimensión espacial y emergieron de la geografía, la arquitectura y la sociología de la escuela de Chicago, haciendo énfasis en aspectos del medio natural, las funciones urbanas, los procesos morfológicos y de construcción urbana, y los modelos espaciales; algunos de sus principales exponentes son Poëte (Almandoz, 2008), Park (1967) o Benévolo (1978, 5 vols.). 
- Otro paradigma se refiere a la ciudad como el ámbito donde ocurren acontecimientos económicos, sociales, políticos y religiosos, en el cual la variable espacial tiene menor valoración; sus autores fundamentales son Weber (1958) y Pirenne (1972), entre otros.

- La tercera de las tendencias tiene un enfoque marcadamente humanístico, que hace énfasis en aspectos culturales y artísticos de la ciudad occidental, sin obviar el elemento espacial; está representada principalmente por el crítico Mumford (1966).

- La escuela de los Anales introdujo una nueva conceptuación y métodos que plantean la "historia-problema" (Febvre, 1982), y articulan el componente geográfico con otras ciencias del hombre para generar una historia total de duraciones diversas en el tiempo; Braudel (1995) es uno de sus más conspicuos autores.

- Una de las corrientes fundamentales que proviene de la historia social y económica fue formulada por Dyos (1968), y se refiere a la conceptuación de la historia urbana como proceso complejo, con menor énfasis en el componente geográfico hasta los años ochenta (Almandoz, 2008); uno de sus autores fundamentales es Briggs (1965), quien se enfoca en la ciudad como proceso complejo, debido a su proveniencia de la historia económica y social.

- Otra aproximación epistemológica insoslayable es la del materialismo histórico, que establece grandes categorías analíticas denominadas 'modos de producción', y que en el ámbito urbano introduce reflexiones sobre las estructuras espaciales y sociales de los estadios de la urbanización, y sobre la producción y consumo del espacio; Korn (1963) y Lefebvre (1976) representan esta posición.

- Valga añadir que, desde los noventa, se han generado diversas tendencias, entre las que destaca la nueva historia urbana. Dos ejemplos que derivaron de los postulados de los Anales y que enfatizan la historia total sobre el evento son la new history británica, preocupada por la interdisciplinariedad, y la nouvelle histoire francesa, interesada en la estructura del discurso (Almandoz, 2008). Un exponente de estas tendencias es el belga Bairoch (1990).

La historiografía urbana de América Latina también presenta aproximaciones epistemológicas propias de su singularidad histórica y espacial, que en algunos casos derivan de los anteriores paradigmas. A partir del pionero y fundamental estudio de Almandoz (2008), podrían delimitarse cuatro grandes vertientes, algunas de ellas entreveradas con la práctica profesional:

- La morfológico-evolucionista, relacionada con los inicios institucionales y académicos del urbanismo latinoamericano, que data de las primeras décadas del siglo $\mathrm{xx}$ y estuvo representada en concepciones subyacentes a las propuestas urbanísticas de Brunner y Della Paolera (Almandoz, 2008).

- Otra de las aproximaciones se relaciona con el proceso de urbanización, dentro de una visión integral de la historia urbana que ha generado periodizaciones y modelos de ciudad. Algunos de sus representantes fundamentales son Hardoy 
(1972), Morse (1975), y en años recientes podría incluirse a Mejía Pavony (2013).

- La aproximación estructuralista de la Escuela de la Dependencia deriva parcialmente del pensamiento marxista y produjo explicaciones sobre la denominada "urbanización dependiente", generó periodizaciones en función de factores económicos y tuvo gran auge en los setenta. Uno de los autores destacados es Rofman (1977).

- Finalmente está la historia sociocultural urbana, que tiene viejas raíces latinoamericanas y aborda la dimensión sociocultural de lo urbano y sus cambios, en tanto que utiliza estrategias interdiscursivas integradoras. Los primeros representantes fueron Romero (1976) y Rama (s.f.), y en años recientes se encuentran Gorelik (1998) y Almandoz (2013).

Este conjunto tan diverso y amplio de interpretaciones sobre la realidad histórica urbana da cuenta de la heterogeneidad epistemológica acerca del fenómeno urbano en la historia. No obstante, la temática sobre la ciudad no es estática y se está ampliando, de manera que se irán constituyendo nuevos paradigmas, como, por ejemplo, los relativos a la sostenibilidad urbana, u otros.

\section{El método histórico urbano}

Como se señaló anteriormente, la historiografía urbana es un subcampo multidisciplinario del saber, de manera que no se puede establecer una práctica metodológica única para el estudio de los procesos históricos urbanos, ya que lo común es la diversidad de los procedimientos y técnicas metodológicas disciplinares. Estas prácticas presentan operaciones comunes al método científico y otras que son propias de cada una de las ciencias asociadas, en las que cada disciplina emplea factores analíticos específicos, instrumentos distintivos y técnicas particulares (Aróstegui, 2001, p. 348) y asume las formas del conocimiento convenientes. En general, los métodos utilizados en las investigaciones de historia urbana comprenden, por una parte, los procedimientos específicos para el estudio de la historia que emplean los historiadores, con énfasis particular en el manejo de las fuentes de información como base material para establecer los hechos históricos y reconstruir la realidad urbana. Por otra parte, están los procesos que siguen los científicos sociales, que -según Singleton et al., (1993) - además de utilizar el método de la historia, hacen énfasis en construcciones conceptuales y teóricas que son demostradas mediante información histórica. A continuación se exponen brevemente las prácticas metodológicas empleadas por los historiadores urbanos.

\section{Los procedimientos de la investigación histórica}

Al igual que otras disciplinas, el conocimiento histórico se construye siguiendo una serie de pasos ordenados de manera sistemática que tienen aceptación más o menos universal, aunque la formulación y el orden pueden variar de autor en autor. Metodólogos de la historia como Topolsky, (1992) y Aróstegui (2001) establecen un conjunto de operaciones que siguen el siguiente orden de general reconocimiento: 
1. Planteamiento del problema en cuanto al tema y el espacio sociohistórico; 2. ${ }^{\circ}$ Formulación de un esquema hipotético o presunción explicativa que intenta responder las preguntas que se fundamentan en el conocimiento acumulado sobre el tema; 3. ${ }^{\circ}$ Localización, acopio, selección y análisis de las fuentes. El proceso de análisis implica la crítica de las fuentes: autenticidad o crítica externa, y fiabilidad o crítica interna; $4 .^{\circ}$ Construcción de los datos a partir del estudio de las fuentes; $5 .^{\circ}$ Establecimiento de los hechos históricos; 6. ${ }^{\circ}$ Desarrollo de la explicación histórica, que significa hacer inteligible el pasado (Le Goff, 1991) a partir de la discusión y generalización (Aróstegui, 2001); y 7. Generación de la síntesis histórica.

La dinámica de la investigación inevitablemente tiende a solapar las anteriores etapas, lo que significa que distintas operaciones pueden realizarse de modo simultáneo en el proceso de investigar. Estos pasos presentan un núcleo central, que se refiere a los documentos y su crítica y a los problemas de síntesis histórica (Veyne, 1984), de manera que en el proceso de investigación se va configurando un problema histórico mediante el movimiento pendular que pasa de los documentos a la síntesis y viceversa.

Un aspecto importante de considerar se refiere a la diferencia existente entre las operaciones metodológicas que presentan un marco conceptual y las que no lo hacen. Al respecto, Singleton et al., (1993) consideran que algunas investigaciones de las ciencias sociales hacen énfasis en construcciones conceptuales y teorías que son demostradas mediante información histórica, en tanto Tuchman (1994) opina que los fenómenos sociales deben ser comprendidos en su contexto histórico. Así, las investigaciones contentivas de un marco teórico introducen las ideas y conceptos que sustentan el desarrollo de la investigación, elaborados sobre la base de teorías preexistentes y otros aportes, de manera que el marco conceptual establece los fundamentos de la interpretación del proceso histórico investigado y de la argumentación empleada para desarrollar la hipótesis de trabajo. Ejemplos de este tipo de investigaciones en obras de historia urbana son el capítulo I de Sjoberg (1974), el análisis de Morse (1975) sobre la teoría del producto principal (stapletheory) de North, la Lección I de Chueca (1980), o el primer capítulo de Almandoz (1997), entre otros.

Con base en lo expuesto sobre el método histórico, en los siguientes apartes se analizan algunas de sus operaciones fundamentales. Nos referimos a las fuentes y a su crítica, a los hechos históricos y a la construcción de la historia. Ellas forman parte de las operaciones metodológicas y son esenciales para entender la especificidad de lo histórico; pero, además, se encuentran entretejidas entre sí a través del proceso de síntesis, como se muestra adelante.

\section{Las fuentes de la historiografía urbana}

Las fuentes son las piezas fundamentales de información sobre el pasado a partir de las cuales se construyen datos que permiten realizar inferencias y establecer los hechos históricos urbanos. Las fuentes han sido clasificadas de diversas maneras, pero en general se dividen en primarias y secundarias. Las primeras son escritos o restos materiales coetáneos con los hechos investigados, que revelan información sobre esos mismos hechos; las secundarias, por su parte, son resultado de un interés 
posterior a los hechos investigados y se materializan en libros, artículos y trabajos académicos. Según Bauer (1957), las fuentes primarias se pueden clasificar de muchas maneras; por ejemplo, escritas, orales, iconográficas, restos o residuos del pasado y tradiciones.

Las fuentes escritas sobre la ciudad abarcan una extensa gama de inscripciones y documentos producidos desde los primeros tiempos de la vida urbana en Mesopotamia o de las estelas maya, cuando los escribas daban cuenta de la vida en las primeras ciudades. Desde esas manifestaciones hasta nuestros tiempos, el volumen y tipo de documentos escritos es inconmensurable: crónicas, informes, leyes y reglamentos, relatos, reportajes, entrevistas, narraciones, memorias, autobiografías, cronologías, epístolas y misivas, entre otros, son expresiones de la diversidad de las fuentes escritas.

Las fuentes materiales sobre la ciudad son numerosas y entre ellas están las edificaciones, calles, plazas e infraestructuras construidas en diversas épocas del pasado, muchas de las cuales podemos observar y utilizar hoy día; también los restos arqueológicos o artefactos dejados por habitantes de aldeas paleolíticas; asimismo, son fuentes los modelos físicos y las maquetas.

Las imágenes comprenden diversas formas de representación cartográfica, como los mapas y planos; las fotografías, que en su evolución técnica y temática muestran la ciudad desde diferentes ángulos; también se encuentran los dibujos, grabados y pinturas realizados por artistas, que datan de la época en que el hombre pintó en las paredes de edificios, como, por ejemplo, el plano ideal de Çatal Hüyük, de hace más de 6.000 ańos. Asimismo, las tarjetas postales, afiches y avisos publicitarios son fuentes iconográficas.

Otro tipo de fuentes son las orales, que abarcan, por ejemplo, las declamaciones de los contadores de historias, las canciones y los protagonistas y testigos de la historia urbana reciente, como, por ejemplo, los habitantes constructores de un barrio popular o un funcionario a cargo de las obras publicas de una ciudad. Asimismo y desde los inicios del siglo xx, las producciones audiovisuales han enriquecido el catálogo de fuentes de la historia urbana, que se acrecientan constantemente con los aportes de las diversas grabaciones orales, las producciones radiales, fílmicas, televisivas y digitales, hasta los modelos virtuales generados en décadas recientes.

Sin embargo, en numerosos casos las fuentes documentales, en sus diversas manifestaciones, deben ser verificadas en su autenticidad y veracidad, operaciones sin las cuales se estaría incurriendo en errores históricos.

\section{La crítica de las fuentes}

En esto consiste la crítica de las fuentes, o conjunto fundamental de operaciones técnicas esenciales en el proceso metodológico de la historia, cuyo objetivo es determinar la autenticidad (crítica externa o erudita) y la determinación del hecho o veracidad (crítica interna) de la información que va a ser empleada en la investigación. La autenticidad se refiere a la determinación del tiempo y el lugar de origen de una fuente concreta, el establecimiento de su autor y a probar si la pieza es genuina o falsa, como lo apunta Bauer (1957). En numerosas investigaciones, el establecimiento de la autenticidad de los documentos, salvo circunstancias excepcionales, 
estriba en la credibilidad de las instituciones que las conservan y en el criterio de confiabilidad primaria. La determinación de la veracidad o credibilidad es el grado de acuerdo entre el mensaje y los hechos. Esta se establece mediante la comparación de datos provenientes de diversas fuentes, por ejemplo los procedentes de diversas instituciones públicas, lo que permite establecer los hechos urbanos con mayor grado de certidumbre. $\mathrm{Al}$ respecto, la publicación periódica de datos seriados, como por ejemplo las memorias gubernamentales, permite realizar un seguimiento de los hechos históricos y corroborar la información. Asimismo, los datos contenidos en las fuentes cartográficas pueden ser analizados mediante el estudio de su evolución temporal.

Una vez realizada la crítica de las fuentes, se procede a construir los hechos históricos urbanos.

\section{Los hechos históricos urbanos}

De acuerdo con Carr (1982), el hecho histórico es un acontecimiento que el investigador considera relevante, de manera que no es un dato "positivo", sino el resultado de una construcción activa cuyo objetivo es transformar la fuente en documento y, luego, constituir esos documentos y hechos históricos en problema (Le Goff, 2001). En tal sentido, el investigador urbano debe evaluar y seleccionar los hechos importantes para su problema de investigación, y descartar los insignificantes.

Por consiguiente, los hechos historiográficos urbanos son construcciones fundamentadas en el heterogéneo y amplio abanico de datos basados en fuentes de información que utilizan los investigadores para producir conocimientos sobre los procesos urbanos. En este sentido, el establecer los hechos históricos plantea operaciones metodológicas directas e indirectas. Las operaciones directas se apoyan en la información de las fuentes que se refieren justamente a ese hecho y que conduce a la interpretación estricta de la huella material que deja un objeto, bien sea un documento, una fotografía, un espacio urbano o un testimonio, que -de acuerdo con Bloch (2012) - se realiza a través de un proceso de simple razonamiento.

Cuando las fuentes disponibles carecen de datos adecuados para proporcionar respuestas a las preguntas planteadas, se formulan operaciones indirectas que son complejas. Para ello se utilizan datos basados en fuentes que no hablan sobre el hecho mismo, pero son indicios que cumplen la función de indicadores.

Algunos procedimientos indirectos que menciona Topolsky (1992, p. 362) son: el geográfico, el comparativo, el regresivo o el de estimaciones estadísticas. En este último caso, la determinación de los indicios y el establecimiento de los hechos urbanos se realiza a través de fases intermedias en las que se emplean distintos recursos metodológicos.

Así, una parte de las investigaciones de historia urbana utiliza los métodos cartográficos de representación planimétrica para construir imágenes derivadas de planos base como los topográficos, con el fin de plasmar hechos sociales y morfológicos ocurridos en el espacio de las ciudades, lo que requiere, según George (1973), de la clasificación, selección, jerarquización y sistematización de los datos. También se aplica el denominado método cuantitativo para establecer mediciones específicas; por ejemplo, datos demográficos a partir de fuentes como los censos de población, 
de manera que es posible estimar la población para fechas sobre las que no existen datos. En este último caso, los investigadores asumen la explicación científica como forma del conocimiento fundamental para construir datos necesarios.

Asimismo, cuando no existen datos directos, se emplea el llamado método comparativo para establecer hechos a partir de inferencias por analogía; por ejemplo, cuando se comparan "series que son similares estructuralmente" (Topolsky, 1992, p. 367), como es el caso de las ciudades coloniales hispanoamericanas.

En ciertos casos, el denominado método regresivo consiste en comparar un determinado espacio urbano en distintos periodos partiendo de fenómenos recientes para reconstruir los hechos más antiguos, como el trazado y el parcelario urbano. Este procedimiento abarca el "método de las permanencias para explicar un hecho urbano" planteado por Aldo Rossi (1982, p. 100), que consiste en aislar las permanencias urbanas que muestran lo que la ciudad ha sido; por ejemplo, los monumentos, los elementos físicos del pasado y los trazados urbanos que se manifiestan en el plano. Estos últimos son "la permanencia más significativa que está dada por las calles y por el plano”, según la teoría de las permanencias de Marcel Poëte, presentada por Rossi (1982, p. 99).

Una vez establecidos los hechos históricos a partir de fuentes que contienen información directa e indirecta, el investigador urbano pasa a una fase en la que, de acuerdo con Aróstegui (2001), se procede a clasificar los hechos con base en categorías conceptuales como las jurídicas, sociales, económicas, espaciales o morfológicas, las que permiten concebir los acontecimientos. Esta fase está relacionada con la siguiente operación, referida a la construcción del texto, donde se efectúa la síntesis histórica.

\section{La construcción en la historiografía urbana}

La construcción de un texto de historia se refiere al proceso de organización de los hechos y de las ideas en función de dar respuesta a las preguntas planteadas en la investigación. Estas operaciones están imbricadas con su forma de transmisión o comunicación a través de un lenguaje coherente, que puede ser el escrito, oral o numérico, y que es denominado discurso o representación histórica. El proceso de exposición organizada de las partes, o discurso, está compuesto por elementos narrativos y analíticos, estos últimos denominados "argumentativos" por Aróstegui (2001), como en el caso de las ciencias sociales. Así, los relatos exponen los acontecimientos secuencialmente con base en una trama comprensible que tiene a la narrativa como componente fundamental (Veyne, 1984). En tanto las monografías analíticas, más cercanas en su estructura a numerosos estudios provenientes de las ciencias sociales, también utilizan la narración como forma de transmitir las partes de la investigación. Por lo tanto, el discurso histórico emplea en mayor o menor medida el lenguaje narrativo. ${ }^{2}$ Una vez expuestos los diversos lenguajes

2 En el discurso histórico reciente la narrativa juega un papel fundamental, tanto en la nueva historia en cuanto decurso de entendimiento que revela aspectos escondidos y conduce la historia a su conclusión (Ricoeur en Almandoz, 2008, p. 184); como en la microhistoria, con la "mayor presencia del punto de vista del investigador en tanto parte intrínseca del recuento histórico" (Almandoz, 2008, p. 185). 
en la construcción de textos, parece evidente la existencia de ciertos vínculos entre las formas del conocimiento y el tipo de discurso empleado; por ejemplo, entre la comprensión y el uso de elementos analíticos en el discurso, o entre la explicación demográfica y el manejo del lenguaje numérico.

Los historiadores de la ciudad, igualmente, integran en su discurso elementos narrativos, descriptivos y analíticos. Ejemplos de los componentes narrativos son las descripciones de ciertos arquitectos sobre la morfología urbana, o el análisis acerca de las transformaciones de algunos componentes urbanos, como los de orden jurídico y socioeconómico en las fases de las ciudades medievales que caracteriza Chueca (1980). Morris (1995, pp. 10-18) a su vez, construye un discurso sintético que combina la descripción de casos, con argumentaciones fundamentadas en elementos analíticos denominados "determinantes de la forma urbana"; por ejemplo, las climáticas en la morfología de ciudades sumerias.

Con relación a la historiografía latinoamericana, autores esenciales -como el historiador Romero (1976) - construyen representaciones que son fundamentalmente narrativas, combinadas con componentes analíticos. Por su parte, el urbanista Almandoz (2013) emplea discursos argumentativos y narrativos basados en conceptos complejos, mediante los cuales analiza los procesos urbanos en varios periodos históricos y explica sus transformaciones.

\section{La temporalidad y las periodizaciones urbanas}

El tiempo es una variable esencial para la historiografía, que es aprehendida y medida como referencia cuantitativa y cualitativa de los acontecimientos humanos y urbanos que ocurren en el tiempo. Para Aróstegui (2001), el análisis de la temporalidad se apoya en tres concepciones: el tiempo cronológico, el tiempo interno y la periodización.

El tiempo cronológico, según Le Goff (1991), es el hilo conductor de los acontecimientos, y se mide a través de los días y años. El tiempo interno tiene que ver con las regularidades y las rupturas en las sociedades y ha sido categorizado por Braudel (1990) en tres duraciones que coexisten: el acontecimiento, de corta duración, denominado episódico; la oscilación cíclica, intercíclica o de coyuntura de los hechos económicos y sociales, que se mide por decenas de ańos; y, finalmente, el tiempo de larga duración o tesis de las estructuras casi inmóviles, desarrolladas en centurias; por ejemplo las ciudades.

Si la cronología es básica para el historiador de la ciudad, la periodización es indispensable para toda forma de comprensión de los procesos urbanos, ya que, según Leff (en Le Goff, 1991), es el instrumento principal de inteligibilidad de los cambios significativos. Si bien la ciudad es considerada una estructura de larga duración, la historiografía urbana occidental ha presentado diversos criterios de periodización de la urbanización, entre los que pueden distinguirse al menos dos vertientes. Por un lado se encuentra la establecida por el científico social Sjoberg (1976), quien agrupa los asentamientos humanos en tres grandes etapas o niveles de organización social, que coexisten actualmente: las aldeas agrícolas formadas con la aparición de la agricultura hace 12.000 ańos; la etapa urbana, comenzada 
hace aproximadamente 5.500 años; y la etapa moderna iniciada a mediados del siglo Xviri con la Revolución Industrial.

Por otra parte y desde la perspectiva morfológica, arquitectos como Benévolo (1978) y Chueca (1980), entre otros, han periodizado la ciudad con base en las épocas de la historia, por ejemplo las ciudades de la antigüedad y las ciudades medievales; las ciudades de la época moderna se han clasificado en renacentistas y barrocas; y las de la era contemporánea corresponden a las ciudades industriales. A su vez, algunos de estos tipos de ciudad se han subdividido en modelos correspondientes a distintas culturas, como por ejemplo la ciudad asiria o helénica en la antigüedad, perspectivas presentadas por Benévolo (1978, vol. 2), Chueca (1980) y Morris (1995), entre otros.

En el contexto latinoamericano, las ciencias sociales, la historiografía y la arquitectura también han generado grandes periodizaciones que siguen criterios de orden político, social, económico, intelectual o morfológico; por ejemplo, Hardoy (1972) ha establecido las siguientes etapas: la ciudad prehispánica, la colonial, la ciudad republicana y la ciudad industrial o masificada.

A su vez, estas etapas también han sido subdivididas en función de criterios diversos: así, el historiador Romero (1976) segmenta la ciudad colonial de acuerdo con aspectos sociales y del poder, y la clasifica en dos etapas: la de la ciudad hidalga y la de la ciudad criolla; por su parte, el economista Singer (1977) divide la ciudad colonial en la fase de la conquista y la fase de la ciudad comercial, que a su vez se extiende al periodo republicano. Desde una perspectiva cultural y profesional, el urbanista Almandoz (2103) inserta nuevas fases a la ciudad republicana, que denomina, primero, la etapa de los programas progresistas y los mitos urbanos, a partir del último tercio del siglo xIx; desde finales del xIX sigue la etapa del arielismo y modernismo o de la Bella Época e higiene; continúa la etapa de la masificación, urbanismo y planes manifiestos, que se inicia en la primera posguerra; finaliza con la etapa del desarrollismo, funcionalismo y planificación, que comienza en la segunda posguerra.

Estas y otras contribuciones demuestran que las periodizaciones son fundamentales, no solo para la caracterización de las estructuras y los fenómenos urbanos en determinadas etapas históricas, sino para la comprensión de las transformaciones urbanas en el tiempo. Ellas parecen darle la razón a Leff en cuanto a que la periodización es el principal instrumento de inteligibilidad de los cambios significativos; entre ellos, los procesos urbanos.

\section{Consideraciones provisionales}

En este ensayo se han expuesto y explorado brevemente algunos de los fundamentos epistemológicos y metodológicos de la historiografía urbana, y en ello se ha presentado una visión novedosa sobre la metodología de la historiografía urbana. A la vez, se ha realizado un aporte al análisis de sus fundamentos epistemológicos a través de una aproximación inédita a las formas del conocimiento de la historiografía urbana. De esta sucinta exploración derivan varias consideraciones. 
La primera es que se reitera el carácter amplio y diverso de la historiografía urbana, primeramente porque los textos estudiados emplean todas las formas conocidas del conocimiento que ya fueron expuestas y, segundamente, debido a la multiplicidad y heterogeneidad de las aproximaciones epistemológicas que pudieron ser trazadas. Así, este breve análisis de los fundamentos constitutivos de la historiografía urbana valida su esencia multidimensional y, por lo tanto, compleja.

La segunda consideración entronca parcialmente con la anterior y se refiere a que, si bien es cierto que la historiografía urbana es un área temática de la historia, también es válido considerarla como una disciplina académica que incorpora a su carácter histórico esencial, los saberes, concepciones y métodos propios de diversas áreas del conocimiento, que confluyen en la descripción, comprensión y explicación del fenómeno urbano en el devenir histórico.

La tercera se refiere a la historiografía urbana latinoamericana, la cual, como parte de la occidental, mantiene su mismo carácter complejo y heterogéneo, pero presenta aproximaciones epistemológicas propias surgidas de la interpretación de su realidad histórica singular, al igual que sucede con las periodizaciones específicas que se han establecido para comprender su peculiar proceso de urbanización. De esta forma, en el caso de la urbanización latinoamericana es posible establecer que algunos de sus fundamentos referidos a las conceptuaciones epistemológicas y de temporalidad, son propios y característicos de su historiografía urbana.

Para finalizar, cabe destacar que estas reflexiones tienen un carácter provisional, dado que se ha partido únicamente de algunas de las obras estatutarias de la historiografía urbana occidental y latinoamericana. A estos ejemplos puntuales se debe añadir la cuantiosa casuística existente. Ello indica que en la historiografía urbana persisten numerosos aspectos por explorar y analizar, y en la medida en que se tome en cuenta esa amplia gama de obras, se enriquecerán los fundamentos de la historiografía urbana expresados en las distintas formas del conocimiento, en los paradigmas o aproximaciones interpretativas y en las operaciones metodológicas de esta compleja área del conocimiento.

\section{Referencias bibliográficas}

Almandoz, A. (1997). Urbanismo europeo en Caracas (1870-1940). Caracas: Equinoccio \& Fundarte.

Almandoz, A. (2008). Entre libros de historia urbana. Caracas: Equinoccio.

Almandoz, A. (2013). Modernización urbana en América Latina. De las grandes aldeas a las metrópolis masificadas. Santiago de Chile: Instituto de Estudios Urbanos y Territoriales (IEUT), Pontificia Universidad Católica de Chile.

Almandoz, A. \& González Casas, L. (1996). Notas sobre historiografía urbana. La visión de la Universidad Simón Bolívar. Urbana, 1(19), 122-126. http://190.169.94.12/ojs/index. $\mathrm{php/rev \_ urb/article/view/8373/8265}$

Alonso, W. (1965). Location and land use. Cambridge, Ma: Harvard University Press. Aróstegui, J. (2001). La investigación histórica, teoría y método. Barcelona: Crítica. 
Bairoch, P. (1990). De Jericó a México. Historia de la urbanización. México, DF: Trillas.

Bauer, W. (1957). Introducción al estudio de la historia. Barcelona: Bosch.

Benévolo, L. (1978). Diseño de la ciudad (vols. 1-5). Barcelona: Gustavo Gili.

Bloch, M. (2012). Introducción a la historia. México, DF: Fondo de Cultura Económica.

Braudel, F. (1990). La historia y las ciencias sociales. Madrid: Alianza Editorial.

Braudel, F. (1995). The Mediterranean and the Mediterranean world in the age of Philip II. (vol. 1). Berkeley, Ca: University of California Press.

Briggs, A. (1965). Victorian cities. New York: Harper \& Row.

Capel, H. (1975). La definición de lo urbano. Estudios geográficos, (138-139), 265-301. http:// www.ub.edu/geocrit/sv-33htm

Capel, H. (2002). La morfología de las ciudades (vol. 1). Barcelona: Ediciones del Serbal.

Carr, E. H. (1982). What is history? London: Penguin Books.

Chueca, F. (1980). Breve historia del urbanismo. Madrid: Alianza Editorial.

Comisión Económica para América Latina y el Caribe (Cepal) (1976). El impacto de la urbanización sobre la sociedad. En G. Germani (comp.), Urbanización, desarrollo y modernización. Un enfoque histórico y comparativo (pp. 278-294). Buenos Aires: Paidós.

Dyos, H. J. (1968). Agenda for Urban Historians. En H. J. Dyos (ed.), The study of Urban History (pp. 1-47). Londres: Edward Arnold.

Febvre, L.(1982). Combates por la historia. Barcelona, España: Ariel.

Gadamer, H. G. (1993). El problema de la conciencia histórica. Madrid: Tecnos.

Gasparini, G. \& Posani, J. P. (1969). Caracas a través de su arquitectura. Caracas: Fundación Fina Gómez.

Germani, G. (1976). La ciudad, el cambio social y la gran transformación. En G. Germani (comp.), Urbanización, desarrollo y modernización. Un enfoque histórico y comparativo (pp. 9-67). Buenos Aires: Paidós.

George, P. (1973). Los métodos de la geografía. Barcelona: Oikos-Tau Ediciones.

Gorelik, A. (1998). La grilla y el parque. Espacio público y cultura urbana en Buenos Aires, 18871936. Buenos Aires: Universidad Nacional de Quilmes.

Guardia, M., Monclús, F. J., \& Oyón, J. (1996). Los atlas de ciudades, entre la descripción y la comparación. En C. Sambricio (ed.), La historia urbana (pp. 109-134). Madrid: Marcial Pons.

Hardoy, J. E. (1972). Las ciudades en América Latina. Buenos Aires: Paidós.

Hernández, R., Fernández, C., \& Baptista, P. (1998). Metodología de la investigación. México, DF: Mc Graw Hill Interamericana Editores.

Jaramillo, S. (1989-1990). El desenvolvimiento de la discusión sobre la urbanización latinoamericana: ¿Hacia un nuevo paradigma de interpretación? En M. Unda (ed.), La investigación urbana en América Latina. Caminos recorridos y por recorrer. Viejos y nuevos temas (vol. 2, pp. 35-74). Quito: Ciudad.

Korn, A. (1963). La historia construye la ciudad. Buenos Aires: Editorial Universitaria de Buenos Aires.

Kuhn, T. (2004). La estructura de las revoluciones cientificas. México, DF: Fondo de Cultura Económica.

Le Goff, J. (1991). Pensar la historia. Barcelona: Paidós.

Le Goff, J. (2001). Prefacio. En M. Bloch, Apología para la historia o el oficio del historiador (pp. 9-33). México, DF: Fondo de Cultura Económica. 
Lefebvre, H. (1976). La revolución urbana. Madrid: Alianza Editorial.

Martindale, D. \& Neuwirth, G. (1958). Prefatory remarks: the theory of the city. En M. Weber, The city (pp. 9-62). Glencoe, IL: The Free Press.

Mejía Pavony, G. (2013). La aventura urbana de América Latina. Madrid: Taurus /Fundación Mapfre.

Morris, A. E. J. (1995). History of urban form, before the Industrial Revolutions. Essex, UK: Longman Scientific \& Technical.

Morse, R. (1975). El desarrollo de los sistemas urbanos en las Américas durante el siglo XIX. En J. E. Hardoy \& R. P. Schaedel (eds.), Las ciudades de América Latina y sus áreas de influencia a través de la historia (pp. 263-290). Buenos Aires: Sociedad Interamericana de Planificación (SIAP).

Mumford, L. (1937). Whatis a city? Architectural Record 82(2), 58-62. http://www. architecturalrecord.com/ext/resources/archives/backissues/1937-11pdf?-1015095600

Mumford, L. (1966). La ciudad en la historia. (vols.1-2). Buenos Aires: Ediciones Infinito.

Negrón, M. (2001). Ciudad y modernidad. Caracas: Ediciones del Instituto de Urbanismo.

Palacios, L. C. (1980). Acerca de la estructura urbana. Urbana, 1 (1), 4-33. http://190.169.94.12/ ojs/index.php/rev_urb/article/view/9027/8881

Park, R. (1967). The city. Suggestions for investigation for human behavior in the urban environment. Chicago, IL: The University of Chicago Press.

Pirenne, H. (1972). Las ciudades de la Edad Media. Madrid: Alianza Editorial.

Rama, A. (s.f.). La ciudad ordenada. Montevideo: Comisión Uruguaya pro Fundación Internacional Ángel Rama.

Rofman, A. (1977). Dependencia, estructura de poder y formación regional en América Latina. Buenos Aires: Siglo Xxi Editores.

Romero, J. L. (1976). Latinoamérica: las ciudades y las ideas. Buenos Aires: Siglo Xxi Editores.

Rossi, A. (1982). La arquitectura de la ciudad. Barcelona: Gustavo Gili.

Sambricio, C. (1996). Introducción. En C. Sambricio (ed.), La historia urbana (pp. 11-14). Madrid: Marcial Pons.

Singer, P. (1977). Campo y ciudad en el contexto histórico latinoamericano. En P. Singer, Economia política de la urbanización (pp. 109-136). México, DF: Siglo Xxi Editores.

Singleton, R., Straits, B., \& Miller S., M. (1993). Approaches to social research. New York: Oxford University Press.

Sjoberg, G. (1974). La ciudad preindustrial. Pasado y presente. Caracas: Universidad Central de Venezuela, Ediciones de la Biblioteca.

Sjoberg, G. (1976). El origen y evolución de las ciudades. En K. Davies (ed.), La ciudad: su crecimiento e impacto en el hombre (pp. 17-27). Madrid: Hermann Blume.

Terán, F. de. (1996). Historia urbana moderna en España. Recuento y acopio de materiales. En C. Sambricio (ed.), La historia urbana (pp. 11-14). Madrid: Marcial Pons.

Topolsky, J. (1992). Metodología de la historia. Madrid: Cátedra.

Tuchman, G. (1994). Historical social science. En N. Denzin \& Y. Lincoln (eds.), Handbook of Qualitative Research (pp. 306-323). Thousand Oaks, ca: Sage Publications.

Veyne, P. (1984). Cómo se escribe la historia. Madrid: Alianza Editorial.

Weber, M. (1958). The City. Glencoe, IL: The Free Press.

Wirth, L. (1968). El urbanismo como modo de vida. Buenos Aires: Ediciones 3. 